\title{
Nonparametric maximum likelihood estimation of the structural mean of a sample of curves
}

\author{
BY DANIEL GERVINI \\ Department of Mathematical Sciences, University of Wisconsin-Milwaukee, \\ 3200 N. Cramer St., Room E490, Milwaukee, Wisconsin 53211, U.S.A. \\ gervini@uwm.edu \\ AND THEO GASSER \\ University of Zürich, Sumatrastrasse 30, CH-8006 Zürich, Switzerland \\ tgasser@ifspm.unizh.ch
}

\section{SUMMARY}

A random sample of curves can be usually thought of as noisy realisations of a compound stochastic process $X(t)=Z\{W(t)\}$, where $Z(t)$ produces random amplitude variation and $W(t)$ produces random dynamic or phase variation. In most applications it is more important to estimate the so-called structural mean $\mu(t)=E\{Z(t)\}$ than the crosssectional mean $E\{X(t)\}$, but this estimation problem is difficult because the process $Z(t)$ is not directly observable. In this paper we propose a nonparametric maximum likelihood estimator of $\mu(t)$. This estimator is shown to be $\sqrt{ } n$-consistent and asymptotically normal under the assumed model and robust to model misspecification. Simulations and a realdata example show that the proposed estimator is competitive with landmark registration, often considered the benchmark, and has the advantage of avoiding time-consuming and often infeasible individual landmark identification.

Some key words: Curve registration; Functional data; Longitudinal data; Phase variation; Time warping.

\section{INTRODUCTION}

Multivariate datasets often consist of discrete observations of continuous curves. A classical example is the longitudinal analysis of growth data. Although the data consist of vectors, classical multivariate techniques that do not take into account the underlying smoothness of the curves are either very inefficient or must resort to strong model assumptions that are dubious in many applications. Ramsay \& Silverman $(1997,2002)$ make a strong case for the nonparametric approach to the statistical analysis of samples of curves.

A characteristic feature of samples of curves, as opposed to samples of arbitrary vectors, is the presence of time variability. Figure 1(a) illustrates this problem well, showing four representative leg-growth velocity curves for boys, vertically shifted for better visualisation. These are raw velocity curves, obtained directly from leg-length data without smoothing. The peaks of maximal pubertal growth occur approximately at 14 years of age, but the exact timing and amplitude vary from person to person. The cross-sectional mean, see Fig. 1(c), is a poor estimate of the average growth velocity, not so much because of its 


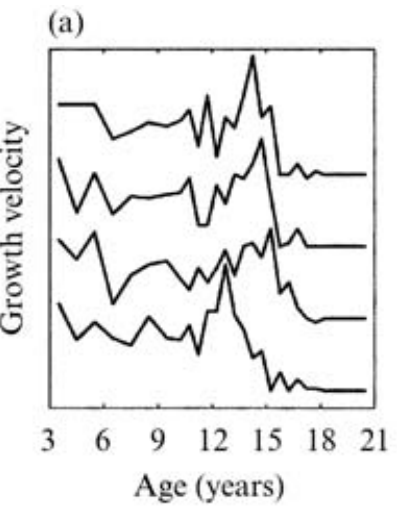

(b)

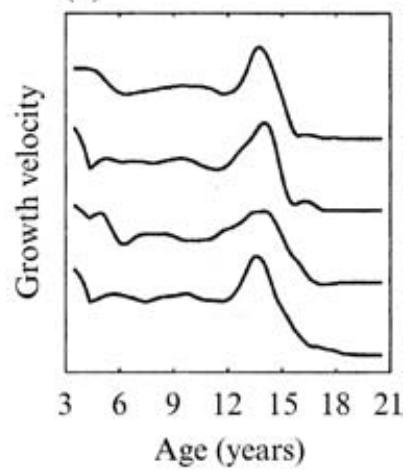

(c)
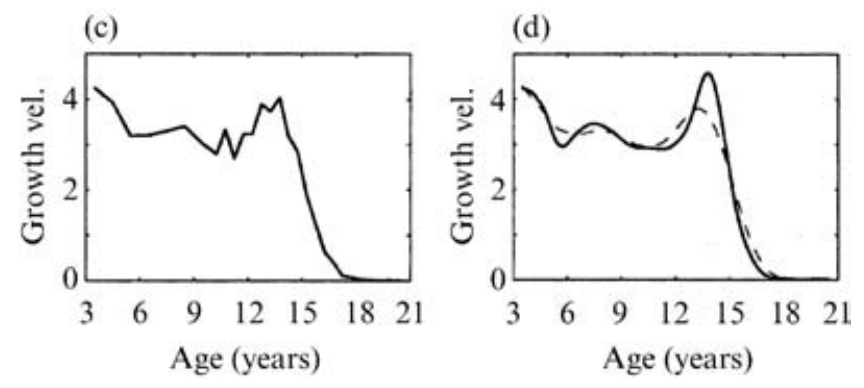

Fig. 1: Leg-growth velocity of boys. (a) Four representative raw sample curves and (b) the corresponding aligned and smoothed curves. (c) Cross-sectional sample mean of growth velocity in $\mathrm{cm} /$ year. (d) Nonparametric maximum likelihood estimator of the structural mean, solid line, and smoothed cross-sectional mean, dashed line, of growth velocity in $\mathrm{cm} /$ year.

roughness, which can be smoothed out with any of the well-known univariate smoothing methods, but mainly because it grossly underestimates the average growth velocity at the pubertal peak, a direct consequence of time variability. Figure 1(b) shows the same sample curves smoothed and aligned using the techniques that will be introduced in this paper, and Fig. 1(d) shows the resulting estimator of mean growth velocity.

Several recent methods for handling time variability involve aligning the curves so as to remove most of the time variability prior to averaging. The method generally considered to be the benchmark is landmark registration (Kneip \& Gasser, 1992). The procedure consists of identifying a set of salient features in all the curves, such as local extrema or zero crossings, monotonically transforming the curves so that the transformed landmarks of each curve coincide with the average landmarks, and then computing the average of the aligned curves. The disadvantage of landmark registration is that a completely automated identification of landmarks is often not possible. The researcher must then identify the landmarks curve by curve, which is infeasible for large datasets. For this reason, alternative methods have been sought; see Silverman (1995), Ramsay \& Li (1998), Kneip et al. (2000), Wang \& Gasser (1999), Rønn (2001) and Gervini \& Gasser (2004). These methods differ greatly in terms of range of applicability, computational complexity and theoretical background.

To make the discussion more formal, we assume that our sample curves are $n$ independent realisations of a compound stochastic process $X(t)=Z\{W(t)\}$, where $Z(t)$ 
produces random amplitude variability and $W(t)$ produces random time variability, and it is therefore assumed to be monotone increasing with probability one. The researcher usually wants to estimate $\mu(t)=E\{Z(t)\}$, the so-called structural mean (Kneip \& Gasser, 1992), rather than the cross-sectional mean $E\{X(t)\}$. Kneip \& Engel (1995) showed that the mean of landmark-registered curves is a consistent estimator of $\mu$ under certain conditions. Consistency, however, holds under the assumption that the number of observations per individual, $m$, goes to infinity and the number of individuals $n$ is held fixed; moreover, the rate of convergence depends on the smoothing bandwidth used to extract the individual landmarks. Consistency of the estimators of Wang \& Gasser (1999) and Gervini \& Gasser (2004) is also proved for $m$ going to infinity and $n$ fixed. However, this type of asymptotics is intrinsically inadequate in most applications, in which individuals are random rather than fixed factors. On the other hand, the articles of Silverman (1995), Ramsay \& Li (1998) and Kneip et al. (2000) do not provide any kind of consistency results for their estimators.

In contrast, the nonparametric maximum likelihood estimator of Rønn (2001) is $\sqrt{ } n$-consistent and asymptotically normal as $n$ goes to infinity and $m$ is fixed; this has been proved by B. Rønn and I. Skovgaard in an unpublished technical report of the Royal Veterinary and Agricultural University, Frederiksberg. This is the relevant type of asymptotics when the number of random curves is large but the number of observations per curve is fixed and relatively small. The human growth data shown in Fig. 1 and analysed later in $\S 7$ are a typical example of this. Rønn's method was derived under the assumption that the warping process is a scalar random shift, which is too simplistic in most applications. For example, the growth curves in Fig. 1(a) have fixed endpoints and present two growth spurts, the mid-growth spurt about age 7 and the pubertal spurt about age 14, whose locations vary independently of each other; obviously, the use of a single random shift for the whole curve is inadequate.

However, the idea of estimating $\mu$ by maximum likelihood is appealing because it avoids individual identification of landmarks. In fact, it avoids estimation of individual parameters altogether, since individual random effects are integrated out rather than estimated. This is why consistency of $\hat{\mu}$ as $n$ goes to infinity is attainable. What we propose in this paper is nonparametric maximum likelihood estimation with more flexible families of warping functions. As by-products, we derive individual predictors for the warping functions $W_{i}(t)$ and the amplitude process $Z_{i}(t)$. Bootstrap methods for constructing confidence bands for $\mu(t)$ are also proposed.

\section{The MAXimum LiKeliHood ESTIMATOR}

As explained in $\S 1$, we will assume that the dataset $\left\{x_{1}, \ldots, x_{n}\right\}$, with $x_{i} \in \mathbb{R}^{m_{i}}$, consists of discrete and noisy realisations of stochastic processes $X_{i}: T \rightarrow \mathbb{R}$, with $T=[a, b]$, so that $x_{i j}=X_{i}\left(t_{i j}\right)+\varepsilon_{i j}$, where $\left\{\varepsilon_{i j}\right\}$ are independent and identically distributed random errors and $\left\{t_{i 1}, \ldots, t_{i m_{i}}\right\} \subset T$ is an input grid that may be different for each individual. The processes $X_{1}(t), \ldots, X_{n}(t)$ are assumed to be independent and identically distributed realisations of a stochastic process $X(t)=Z\{W(t)\}$. The warping process will be parametrically modelled as $W(t)=g(t, \theta)$, where $g$ is a fixed, known function, monotone increasing in $t$ for every $\theta$. The parameter $\theta \in \mathbb{R}^{p}$ will be considered to be an unobservable random effect. Possible families of warping functions $g$ and distributions for $\theta$ will be discussed in $\S 3$. 
For the amplitude component a reasonable model would be

$$
Z(t)=\mu(t)+\sum_{k=1}^{q} \xi_{k} \phi_{k}(t)
$$

where $\mu$ and $\left\{\phi_{k}\right\}$ are fixed unknown functions, $\int_{a}^{b} \phi_{k}(t) \phi_{l}(t) d t=\delta_{k l}$, and $\left\{\xi_{k}\right\}$ are independent zero-mean random variables with finite variances. This is a truncation of the Karhunen-Loève decomposition $Z(t)=\mu(t)+\sum_{k=1}^{\infty} \xi_{k} \phi_{k}(t)$, which holds for any square-integrable stochastic process. Unfortunately, simultaneous maximum likelihood estimation of $\mu$ and the components $\left\{\phi_{k}\right\}$ is very complicated. For simplicity, we will derive the maximum likelihood estimator of $\mu$ for a model without variance components. It is shown later, by simulations and example, that this maximum likelihood estimator provides good estimates of $\mu$ even under the general variance-component model (1).

As working model, then, let us assume the mean-plus-error model

$$
x_{i j}=\mu\left\{g\left(t_{i j}, \theta_{i}\right)\right\}+\varepsilon_{i j}, \quad \varepsilon_{i j} \sim \mathscr{N}\left(0, \sigma^{2}\right),
$$

with $\theta_{i}$ and $\varepsilon_{i j}$ independent. Under this model, $x_{i} \mid \theta_{i} \sim \mathscr{N}\left[\mu\left\{g\left(t_{i}^{*}, \theta_{i}\right)\right\}, \sigma^{2} I_{m_{i}}\right]$, where $t_{i}^{*}=\left(t_{i 1}, \ldots, t_{i m_{i}}\right)^{\mathrm{T}}$; here and in the rest of the paper, evaluation of a univariate function at a vector is understood in a componentwise sense. The loglikelihood function is

$$
L\left(\mu, \sigma^{2}\right)=\sum_{i=1}^{n} \log \int f\left(x_{i} \mid \theta ; \mu, \sigma^{2}\right) f(\theta) d \theta,
$$

where $f\left(x_{i} \mid \theta ; \mu, \sigma^{2}\right)$ denotes the conditional density of $x_{i}$ given $\theta_{i}=\theta$ and $f(\theta)$ is the density of $\theta$.

The estimating equation for $\hat{\sigma}^{2}$ is easily derived. Since

$$
\frac{\partial f\left(x_{i} \mid \theta_{i} ; \mu, \sigma^{2}\right)}{\partial\left(\sigma^{2}\right)}=\left[-\frac{m_{i}}{2 \sigma^{2}}+\frac{\left\|x_{i}-\mu\left\{g\left(t_{i}^{*}, \theta_{i}\right)\right\}\right\|^{2}}{2\left(\sigma^{2}\right)^{2}}\right] f\left(x_{i} \mid \theta_{i} ; \mu, \sigma^{2}\right),
$$

we have

$$
\frac{\partial L\left(\mu, \sigma^{2}\right)}{\partial\left(\sigma^{2}\right)}=\sum_{i=1}^{n} \frac{1}{f\left(x_{i} ; \mu, \sigma^{2}\right)} \int\left[-\frac{m_{i}}{2 \sigma^{2}}+\frac{\left\|x_{i}-\mu\left\{g\left(t_{i}^{*}, \theta\right)\right\}\right\|^{2}}{2\left(\sigma^{2}\right)^{2}}\right] f\left(x_{i} \mid \theta ; \mu, \sigma^{2}\right) f(\theta) d \theta,
$$

where $f\left(x_{i} ; \mu, \sigma^{2}\right)$ is the marginal density of $x_{i}$. Since $\partial L\left(\hat{\mu}, \hat{\sigma}^{2}\right) / \partial\left(\sigma^{2}\right)=0$, we obtain the following fixed-point expression:

$$
\hat{\sigma}^{2}=\frac{1}{\sum_{i=1}^{n} m_{i}} \sum_{i=1}^{n} \int\left\|x_{i}-\hat{\mu}\left\{g\left(t_{i}^{*}, \theta\right)\right\}\right\|^{2} f\left(\theta \mid x_{i} ; \hat{\mu}, \hat{\sigma}^{2}\right) d \theta .
$$

An estimating equation for $\hat{\mu}$ can be derived using Gateaux differentials. Let $\mathscr{M}$ be the parameter space of $\mu$, which is a linear subspace of $\mathscr{L}^{\infty}(T)$, the space of bounded measurable functions $T \rightarrow \mathbb{R}$. Since $\hat{\mu}$ maximises $L\left(\mu, \hat{\sigma}^{2}\right)$ for all $\mu \in \mathscr{M}$, the directional $\operatorname{loglikelihood} L\left(\hat{\mu}+t h, \hat{\sigma}^{2}\right)$ is maximised at $t=0$ for every $h \in \mathscr{M}$. Then

$$
\left.\frac{d}{d t} L\left(\hat{\mu}+t h, \hat{\sigma}^{2}\right)\right|_{t=0}=0
$$

for all $h \in \mathscr{M}$. After straightforward algebra we obtain

$$
\left.\frac{d}{d t} L\left(\hat{\mu}+t h, \hat{\sigma}^{2}\right)\right|_{t=0}=\frac{1}{\hat{\sigma}^{2}} \sum_{i=1}^{n} \int\left[x_{i}-\hat{\mu}\left\{g\left(t_{i}^{*}, \theta\right)\right\}\right]^{\mathrm{T}} h\left\{g\left(t_{i}^{*}, \theta\right)\right\} f\left(\theta \mid x_{i} ; \hat{\mu}, \hat{\sigma}^{2}\right) d \theta .
$$


Let $w_{i j}\left(. \mid x_{i} ; \mu, \sigma^{2}\right)$ be the conditional density of $g\left(t_{i j}, \theta\right)$ given $x_{i}$. Then

$$
\int\left[x_{i}-\hat{\mu}\left\{g\left(t_{i}^{*}, \theta\right)\right\}\right]^{\mathrm{T}} h\left\{g\left(t_{i}^{*}, \theta\right)\right\} f\left(\theta \mid x_{i} ; \hat{\mu}, \hat{\sigma}^{2}\right) d \theta=\sum_{j=1}^{m_{i}} \int\left\{x_{i j}-\hat{\mu}(s)\right\} w_{i j}\left(s \mid x_{i} ; \hat{\mu}, \hat{\sigma}^{2}\right) h(s) d s,
$$

so that (4) is equivalent to

$$
\int k_{n}\left(s ; \hat{\mu}, \hat{\sigma}^{2}\right) h(s) d s=0
$$

for all $h \in \mathscr{M}$, where

$$
k_{n}\left(s ; \mu, \sigma^{2}\right)=\frac{1}{\sigma^{2}} \sum_{i=1}^{n} \sum_{j=1}^{m_{i}}\left\{x_{i j}-\mu(s)\right\} w_{i j}\left(s \mid x_{i} ; \mu, \sigma^{2}\right) .
$$

Estimating equations (3) and (5) are asymptotically unbiased, since for the true parameters $\left(\mu, \sigma^{2}\right)$ we have

$$
\begin{aligned}
E\left[\left\{x_{i j}-\mu(s)\right\} w_{i j}\left(s \mid x_{i} ; \mu, \sigma^{2}\right)\right] & =\int\left\{x_{j}-\mu(s)\right\} w_{i j}\left(s \mid x ; \mu, \sigma^{2}\right) f\left(x ; \mu, \sigma^{2}\right) d x \\
& =f_{g\left(t_{i j}, \theta\right)}(s) \int\left\{x_{j}-\mu(s)\right\} f_{x \mid g\left(t_{i j}, \theta\right)}\left(x \mid s ; \mu, \sigma^{2}\right) d x \\
& =0,
\end{aligned}
$$

for all $s \in T$, and

$$
E\left(E\left[\left\|x_{i}-\mu\left\{g\left(t_{i}^{*}, \theta\right)\right\}\right\|^{2} \mid x_{i}\right]\right)=E\left(E\left[\left\|x_{i}-\mu\left\{g\left(t_{i}^{*}, \theta\right)\right\}\right\|^{2} \mid \theta\right]\right)=m_{i} \sigma^{2} .
$$

The estimating equation (5) for $\hat{\mu}$ is not very useful as it is, but for certain parametric spaces it is possible to derive more explicit equations. For example, if $\mathscr{M}$ is the space of continuous functions $\mathscr{C}(T)$, then (5) holds if and only if $k_{n}\left(s ; \hat{\mu}, \hat{\sigma}^{2}\right)=0$ almost everywhere in $T$, which implies that

$$
\hat{\mu}(s)=\frac{\sum_{i=1}^{n} \sum_{j=1}^{m_{i}} x_{i j} w_{i j}\left(s \mid x_{i} ; \hat{\mu}, \hat{\sigma}^{2}\right)}{\sum_{i=1}^{n} \sum_{j=1}^{m_{i}} w_{i j}\left(s \mid x_{i} ; \hat{\mu}, \hat{\sigma}^{2}\right)}
$$

almost everywhere in $T$. However, the space $\mathscr{C}(T)$ is too large to provide reasonably smooth estimates of $\mu$. Moreover, there is no guarantee that $L\left(\mu, \hat{\sigma}^{2}\right)$ attains a maximum in $\mathscr{C}(T)$. For practical and theoretical reasons, discussed in $\S 5$ below and in the technical report by Rønn and Skovgaard mentioned earlier, $\mathscr{M}$ must be restricted to be a compact subspace of $\mathscr{C}(T)$. Since $L\left(\mu, \hat{\sigma}^{2}\right)$ is continuous in $\mu$, it attains a maximum in any compact space $\mathscr{M}$.

Equation (6) has an interesting intuitive interpretation. At each $s, \hat{\mu}(s)$ is a weighted average of $\left\{x_{i j}\right\}$, where $w_{i j}\left(s \mid x_{i} ; \hat{\mu}, \hat{\sigma}^{2}\right)$ puts more weight on those $x_{i j}$ 's for which $g\left(t_{i j}, \theta\right)$ is expected to be close to $s$ and $x_{i j}$ is not far from its current expected value $\hat{\mu}\left\{g\left(t_{i j}, \theta\right)\right\}$. Thus, (6) provides a sort of automatic curve alignment and smoothing, since the weights are smooth functions of $s$. Note that, following this intuition, we can use

$$
\hat{Z}_{i}(t)=\frac{\sum_{j=1}^{m_{i}} x_{i j} w_{i j}\left(t \mid x_{i} ; \hat{\mu}, \hat{\sigma}^{2}\right)}{\sum_{j=1}^{m_{i}} w_{i j}\left(t \mid x_{i} ; \hat{\mu}, \hat{\sigma}^{2}\right)}
$$

as estimates of the registered curves $Z_{i}(t)=X_{i}\left\{W_{i}^{-1}(t)\right\}$. 
Estimating equations similar to (4) can be derived for the mean and the components of the general variance-component model (1). However, explicit estimating equations like (6) or even (5) cannot be obtained, so that the estimators have to be numerically computed using, for instance, a rather cumbersome functional Newton-Raphson method (Luenberger, 1969, Ch. 10). A more practical compromise is to estimate $\mu$ with the maximum likelihood estimator of the mean-plus-error model derived above, and then estimate the factors $\left\{\phi_{k}\right\}$ using the principal components of the registered functions $\left\{\hat{Z}_{i}\right\}$, as in Ramsay \& Silverman (1997, Ch. 6).

Estimation of the individual effects $\left\{\theta_{i}\right\}$ may also be of interest in some situations, as we will see in $\S 7$. This can be done with the conditional expectation $E\left(\theta \mid x_{i} ; \hat{\mu}, \hat{\sigma}^{2}\right)$ or with the conditional mode $\arg \max f\left(\theta \mid x_{i} ; \hat{\mu}, \hat{\sigma}^{2}\right)$. The conditional mode estimator has an interesting interpretation: since

$$
\arg \max f\left(\theta \mid x_{i} ; \hat{\mu}, \hat{\sigma}^{2}\right)=\arg \min \left(\frac{1}{2 \hat{\sigma}^{2}} \sum_{j=1}^{m_{i}}\left[x_{i j}-\hat{\mu}\left\{g\left(t_{i j}, \theta\right)\right\}\right]^{2}-\log \{f(\theta)\}\right),
$$

this is a penalised least squares estimator, with a penalty term $-\log \{f(\theta)\}$ that shrinks $\hat{\theta}_{i}$ towards the mode of $f(\theta)$. Except for the penalty term, this is similar to the Procrustes registration method of Silverman (1995), which minimises the sum of squares with respect to both parameters $\mu$ and $\theta$. Procrustes registration has a tendency to 'overwarp' the data, producing deformed estimates of $\mu$; see the example of Ramsay \& Silverman (2002, p. 113). To some extent this is a problem of all registration methods that estimate $\mu$ simultaneously with the individual effects. Our method avoids this problem by estimating $\mu$ independently of the $\theta_{i}$ 's.

\section{WARPING MODEL}

In $\S 2$ we derived the maximum likelihood estimator $\hat{\mu}$ for a generic warping function $g(t, \theta)$ and a generic distribution $f(\theta)$ of the random effect. For a successful practical implementation of the maximum likelihood estimator, it is important to specify a warping model that is versatile enough but does not have too many parameters; note that equations (3) and (5) involve multi-dimensional integrals in $\theta$.

One possibility is to take $g(t, \theta)$ as a linear combination of $I$-splines with fixed knots (Ramsay, 1988), where $\theta$ is the vector of basis coefficients. Another possibility is to take $g(t, \theta)=a+(b-a) c(t, \theta) / c(b, \theta)$, where $c(t, \theta)=\int_{a}^{t} e^{w(s, \theta)} d s, w(s, \theta)$ is a linear combination of $B$-splines with fixed knots and $\theta$ is again the vector of basis coefficients (Ramsay, 1998). The problem with both of these models is that it is unclear what a reasonable distribution for $\theta$ might be. Moreover, it may be necessary to use a large number of basis functions to obtain enough model flexibility, which complicates the computation of $\hat{\mu}$.

What we propose is to take $\theta$ as a vector of knots, rather than basis coefficients. Intuitively, we may think of $\theta$ as a vector of 'hidden landmarks'. With this interpretation, a reasonable family of distributions for $\theta$ is the truncated normal, with density

$$
f(\theta) \propto \prod_{k=1}^{p} \frac{1}{\tau_{k}} \varphi\left(\frac{\theta_{k}-\theta_{0 k}}{\tau_{k}}\right) \square\left\{a<\theta_{1}<\ldots<\theta_{p}<b\right\},
$$

with $\theta_{01}<\ldots<\theta_{0 p}$. The $\theta_{0 k}$ 's can be associated with salient features of $\mu$, such as peaks and troughs, which in many practical situations will provide a good fit even with a small 
dimension $p$. For example, for the growth curves in Fig. 1 we use a two-dimensional $\theta$, where each coordinate is associated with a growth spurt. The actual form of $g(t, \theta)$ is not so important as long as the monotonicity in $t$ is ensured and the following identifiability conditions are satisfied: $g(a, \theta)=a, g(b, \theta)=b$ and $g\left(\theta_{k}, \theta\right)=\theta_{0 k}$, for $k=1, \ldots, p$. In $\S \S 6$ and 7 we use shape-preserving cubic polynomial interpolation (Fritsch \& Carlson, 1980), as implemented in the Matlab function pchip.

The unknown parameters $\theta_{0}$ and $\tau$ could, in principle, be incorporated in the likelihood function and estimated together with $\mu$ and $\sigma^{2}$, but in practice this is very time consuming. A workable alternative is to try a few values of $\theta_{0}$ and $\tau$ suggested by visual inspection of the data, and keep those with largest likelihood. In our experience, this approach works well in practice because the maximum likelihood estimator is robust to misspecification of $f(\theta)$; see $\S \S 6$ and 7 .

A more refined way of determining $p, \theta_{0}$ and $\tau$ is by means of the 'structural intensity' method of Gasser \& Kneip (1995). This method consists of computing a nonparametric density estimator of the number of local maxima; the modes of the density reveal the most important landmarks and their approximate distributions. This method only requires identification of the set of local maxima for each curve, which can be done in a fully automated way, as opposed to landmark registration, which requires specific identification of local maxima and usually cannot be carried out without human interaction. For example, a given growth curve may have more than just two local maxima, because of undersmoothing or otherwise; whereas landmark registration requires precise identification of the growth spurts among these local maxima, the structural intensity method only requires identification of all the local maxima.

\section{Computational aspects}

The estimators $\hat{\mu}$ and $\hat{\sigma}^{2}$ can be iteratively computed using the fixed-point expressions (3) and (6). As initial estimators, the simplest choices are $\hat{\sigma}^{2(0)}=\sum_{i=1}^{n} \sum_{j=1}^{m_{i}}\left(x_{i j}-\bar{x}\right)^{2} / n m$ and $\hat{\mu}^{(0)}(t)=\sum_{i=1}^{n} \tilde{x}_{i}(t) / n$, where $\tilde{x}_{i}(t)$ is obtained from $x_{i 1}, \ldots, x_{i m_{i}}$ by interpolation; we use piecewise cubic interpolation. A potential problem with using equation (6) to update the estimate of $\mu$ is that the algorithm may not converge; remember that estimating equation (5) is always satisfied by $\hat{\mu}$, but this is not necessarily the case with equation (6). There are algorithms with guaranteed convergence, such as the steepest ascent algorithm (Luenberger, 1969, Ch. 10), that defines $\hat{\mu}^{(k)}(t):=\hat{\mu}^{(k-1)}(t)+\alpha_{k} k_{n}\left(t ; \hat{\mu}^{(k-1)}, \hat{\sigma}^{2(k-1)}\right)$ where the step $\alpha_{k}$ is chosen to maximise the likelihood function in the direction of $k_{n}\left(s ; \hat{\mu}^{(k-1)}, \hat{\sigma}^{2(k-1)}\right)$. However, finding the optimal step $\alpha_{k}$ involves many recomputations of the likelihood function and is very time consuming. We think it is more practical to use the reweighting algorithm suggested by equation (6). When this algorithm converges, it finds a solution of (5) and thus a stationary point of the loglikelihood function. We have used this algorithm for all simulations and data analyses in this paper and have not found any convergence problems.

The hardest part to implement efficiently is the computation of the $p$-dimensional integrals involved in equations (3) and (6). We use Monte Carlo integration: a random sample $\left\{\theta^{(1)}, \ldots, \theta^{(N)}\right\}$ is generated from $f(\theta)$ and, for instance, $f\left(x_{i} ; \hat{\mu}, \hat{\sigma}^{2}\right)=\int f\left(x_{i} \mid \theta ; \hat{\mu}, \hat{\sigma}^{2}\right) f(\theta) d \theta$ is approximated by $\hat{f}^{(N)}\left(x_{i}\right):=\sum_{l=1}^{N} f\left(x_{i} \mid \theta^{(l)} ; \hat{\mu}, \hat{\sigma}^{2}\right) / N$. The other integrals are estimated in a similar way. 
The computation of $w_{i j}\left(s \mid x_{i} ; \hat{\mu}, \hat{\sigma}^{2}\right)$, on the other hand, requires a more careful approach. Since

$$
\begin{aligned}
w_{i j}\left(s \mid x_{i} ; \hat{\mu}, \hat{\sigma}^{2}\right) & =\frac{d}{d s} \int \square\left\{g\left(t_{i j}, \theta\right) \leqslant s\right\} f\left(\theta \mid x_{i} ; \hat{\mu}, \hat{\sigma}^{2}\right) d \theta \\
& =\frac{d}{d s} \int \square\left\{g\left(t_{i j}, \theta\right) \leqslant s\right\} \frac{f\left(x_{i} \mid \theta ; \hat{\mu}, \hat{\sigma}^{2}\right)}{f\left(x_{i} ; \hat{\mu}, \hat{\sigma}^{2}\right)} f(\theta) d \theta,
\end{aligned}
$$

we use a kernel-smoothed Monte Carlo integral:

$$
\hat{w}_{i j}^{(N, \lambda)}\left(s \mid x_{i}\right):=\frac{1}{N} \sum_{l=1}^{N} \frac{1}{\lambda} K\left\{\frac{g\left(t_{i j}, \theta^{(l)}\right)-s}{\lambda}\right\} \frac{f\left(x_{i} \mid \theta^{(l)} ; \hat{\mu}, \hat{\sigma}^{2}\right)}{\hat{f}^{(N)}\left(x_{i}\right)} .
$$

As $K(t)$ we take the Epanechnikov kernel $K(t)=0 \cdot 75\left(1-t^{2}\right) \llbracket\{|t| \leqslant 1\}$, and as tentative $\lambda$ we take the average oversmoothing bandwidth (Wand \& Jones, 1995, p. 61),

$$
\lambda=\left(\frac{243 c_{1}}{35 c_{2}^{2} N}\right)^{1 / 5} \frac{1}{\sum_{i=1}^{n} m_{i}} \sum_{i=1}^{n} \sum_{j=1}^{m_{i}} s_{i j}
$$

where $s_{i j}$ is the sample standard deviation of $\left\{g\left(t_{i j}, \theta^{(1)}\right), \ldots, g\left(t_{i j}, \theta^{(N)}\right)\right\}, c_{1}=\int K^{2}(x) d x$ and $c_{2}=\int x^{2} K(x) d x$. In particular, for the Epanechnikov kernel we have $c_{1}=\frac{3}{5}$ and $c_{2}=\frac{1}{5}$.

At this point it is important to remark that the maximum likelihood estimator itself does not depend on any bandwidths. If a closed expression for $w_{i j}\left(s \mid x_{i} ; \mu, \sigma^{2}\right)$ existed, it would not be necessary to use the smoother $\hat{w}_{i j}^{(N, \lambda)}\left(s \mid x_{i}\right)$. It turns out, however, that the conditional density $w_{i j}$ never has a closed expression in practice, and must be estimated. The choice of $\lambda$ will determine the smoothness of $\hat{w}_{i j}^{(N, \lambda)}\left(s \mid x_{i}\right)$ and this, in turn, will determine the smoothness of $\hat{\mu}$. In our experience, (11) provides a reasonable bandwidth or at least a good initial guess; a plot of $\hat{\mu}$ will clearly tell the user when a smaller or a larger bandwidth is advisable.

\section{Asymptotics AND INFERENCE}

In this section we prove that the maximum likelihood estimator of $\mu$ is consistent and asymptotically normal as the number of curves $n$ goes to infinity. Let us assume that $\left\{x_{1}, \ldots, x_{n}\right\}$ are independent and identically distributed, so that $m_{i}=m$ and the input grids $\left\{t_{i 1}, \ldots, t_{i m}\right\}$ are the same for all $i$. For simplicity of notation, we will also assume that the error variance $\sigma^{2}$ is known, but it is clear that Theorem 1 can be extended to simultaneous estimation of $\mu$ and $\sigma^{2}$ in a straightforward manner.

Given $x \in \mathbb{R}^{m}$, let $\ell_{x}(\mu)=\log f(x ; \mu)$. The maximum likelihood estimator $\hat{\mu}$ maximises $L_{n}(\mu):=E_{n}\left\{\ell_{x}(\mu)\right\}$, where $E_{n}$ denotes expectation with respect to the empirical measure. Then $\hat{\mu}$ always exists if $\mathscr{M}$ is compact, because $L_{n}(\mu)$ is continuous; it is Fréchet differentiable, as shown in Theorem 2 in the Appendix, and Fréchet differentiability implies continuity (Luenberger, 1969, p. 173). Let $L_{0}(\mu):=E_{0}\left\{\ell_{x}(\mu)\right\}$ be the asymptotic loglikelihood function, where $E_{0}$ is the expectation under the mean-plus-error model (2) with parameter $\mu_{0}$. If model (2) is identifiable, then $\mu_{0}$ is the unique maximiser of $L_{0}(\mu)$; see the proof of part (i) of Theorem 1. The assumption that model (2) is identifiable is clearly necessary for consistency. A simple modification of the identifiability proof of 
Gervini \& Gasser (2004) shows that model (2) is identifiable provided $\mu$ is piecewise strictly monotone, i.e. without 'flat' parts, and the warping model $g(t, \theta)$ is identifiable; this is true of the cubic spline model proposed in $\S 3$. For a precise definition of the supremum norm, tensor product and covariance functional used in the following theorem, see the Appendix.

TheOREM 1. If $\mathscr{M}$ is compact and model (2) is identifiable, then the following hold.

(i) (Strong consistency). We have $\operatorname{pr}\left\{\lim _{n \rightarrow \infty}\left\|\hat{\mu}-\mu_{0}\right\|=0\right\}=1$.

(ii) (Asymptotic normality). If $D$ denotes the differential, let $\mathscr{T}:=E_{0}\left\{D \ell_{x}\left(\mu_{0}\right) \otimes D \ell_{x}\left(\mu_{0}\right)\right\}$, which coincides with $-E_{0}\left\{D^{2} \ell_{x}\left(\mu_{0}\right)\right\}$ under model (2). Then $\sqrt{ } n\left(\hat{\mu}-\mu_{0}\right)$ converges in distribution to a Gaussian process with mean zero and covariance functional $\mathscr{T}^{-1}$ in the space $(\mathscr{M},\|\cdot\|)$.

The strong consistency of $\hat{\mu}$ in the supremum norm implies that $\hat{\mu}(t) \rightarrow \mu_{0}(t)$ almost surely for all $t \in T$. The asymptotic normality of $\sqrt{ } n\left(\hat{\mu}-\mu_{0}\right)$ as a stochastic element of $(\mathscr{M},\|\|$.$) implies that the finite-dimensional projections are asymptotically normal in$ the classical multivariate sense; that is, given an arbitrary vector $t^{*}=\left(t_{1}, \ldots, t_{M}\right)$, $\sqrt{ } n\left\{\hat{\mu}\left(t^{*}\right)-\mu_{0}\left(t^{*}\right)\right\}$ converges in distribution to an $M$-variate Normal distribution with covariance matrix explicitly computable from the covariance functional $\mathscr{T}$. In principle, asymptotic confidence bands for $\mu_{0}$ could be derived from this result, but we have found that such bands tend to be too narrow in practice, having finite-sample coverage levels much smaller than the nominal asymptotic levels. For that reason, we see Theorem 1 mainly as a qualitative result that shows that the nonparametric maximum likelihood estimator is able to attain the parametric consistency rate $n^{-\frac{1}{2}}$.

Although Theorem 1, as given above, applies only to the maximum likelihood estimator for the mean-plus-error model (2), a similar result can be obtained for the maximum likelihood estimator of the general variance-component model (1). However, for the reasons indicated in $\S 2$, we think that the latter estimator is impractical. The simulations and the example in $\$ \S 6$ and 7 show that the maximum likelihood estimator of the meanplus-error model does not have a much larger bias under the general variance-component model, so that bootstrap methods based on this estimator can be used for inference under the more general model.

To construct confidence bands for $\mu$ we propose two bootstrap procedures. The simplest one is based on the so-called wild bootstrap; take $B$ bootstrap samples $\left\{x_{1}^{*}, \ldots, x_{n}^{*}\right\}$ from the sample $\left\{x_{1}, \ldots, x_{n}\right\}$, find the corresponding maximum likelihood estimates $\left\{\hat{\mu}_{1}^{*}(t), \ldots, \hat{\mu}_{B}^{*}(t)\right\}$, and construct confidence bands for $\mu$ using the empirical percentiles of this sample.

The second method, which we call model-based bootstrap, consists of the following steps.

Step 1 . Find the maximum likelihood estimates $\hat{\mu}$ and $\hat{\sigma}^{2}$, individual predictors $\left\{\hat{\theta}_{i}\right\}$ and registered curves $\left\{\hat{Z}_{i}(t)\right\}$, as defined in (7).

Step 2. Using the spectral decomposition of the covariance matrix of $\left\{\hat{Z}_{i}(t)\right\}$, find estimates of the components $\left\{\hat{\phi}_{k}\right\}$ and the individual scores $\left\{\hat{\xi}_{i k}\right\}$ of the variance-component model (1), and choose the number of components $q$. Define the residuals

$$
\hat{\varepsilon}_{i j}=x_{i j}-\hat{\mu}\left\{g\left(t_{j}, \hat{\theta}_{i}\right)\right\}-\sum_{k=1}^{q} \hat{\xi}_{i k} \hat{\phi}_{k}\left\{g\left(t_{j}, \hat{\theta}_{i}\right)\right\}
$$


Step 3. Repeat the following $B$ times.

(a) Take independent bootstrap samples $\left\{\hat{\theta}_{i}^{*}\right\}$ from $\left\{\hat{\theta}_{i}\right\},\left\{\left(\hat{\xi}_{i 1}^{*}, \ldots, \hat{\xi}_{i q}^{*}\right)\right\}$ from $\left\{\left(\hat{\xi}_{i 1}, \ldots, \hat{\xi}_{i q}\right)\right\}$, and $\left\{\hat{\varepsilon}_{i j}^{*}\right\}$ from $\left\{\hat{\varepsilon}_{i j}\right\}$. Define the pseudo-observations

$$
x_{i j}^{*}=\hat{\mu}\left\{g\left(t_{j}, \hat{\theta}_{i}^{*}\right)\right\}+\sum_{k=1}^{q} \hat{\xi}_{i k}^{*} \hat{\phi}_{k}\left\{g\left(t_{j}, \hat{\theta}_{i}^{*}\right)\right\}+\hat{\varepsilon}_{i j}^{*} .
$$

(b) Find the maximum likelihood estimate $\hat{\mu}^{*}$ for the bootstrapped dataset $\left\{x_{1}^{*}, \ldots, x_{n}^{*}\right\}$.

Step 4. Construct confidence bands for $\mu$ using the empirical percentiles of $\left\{\hat{\mu}_{1}^{*}(t), \ldots, \hat{\mu}_{B}^{*}(t)\right\}$.

As a referee pointed out, model-based bootstrap has an advantage over wild boostrap when the curves are observed at different time points: the model-based approach preserves the original time points, while the wild approach will result in some time points being observed multiple times and others not at all, which can give very poor results.

\section{Simulations}

\subsection{Monte Carlo scenarios}

In this section we study by simulation the finite-sample performance of the maximum likelihood estimator and of the bootstrap confidence bands. In particular, we compare the performance of maximum likelihood registration with the two most commonly used methods, landmark registration and continuous monotone registration (Ramsay \& Li, 1998), under different models of amplitude and time variability.

The variance-component model (1) is very general and includes very many special cases. Here we focus on a few nontrivial situations in which dealing with time variability is problematic and the advantages or disadvantages of different methods are easy to see.

Therefore, as structural mean $\mu(t)$ we took a function with three prominent landmarks, two peaks and a trough; see Fig. 2. To be specific, $\mu(t)=\beta_{3}(t)-\beta_{4}(t)+\beta_{5}(t)$, where $\beta_{1}(t), \ldots, \beta_{7}(t)$ are the cubic $B$-spline basis functions in $[0,1]$ with knots $\{0 \cdot 4,0 \cdot 5,0 \cdot 6\}$. Samples were generated from the mean-plus-error model (2) and also from the variancecomponent model (1) with $q=1$ and $\phi_{1}(t)=\beta_{4}(t)$, shown in Fig. 2. As input grid we took $m=30$ equispaced points in $[0,1]$. The component $\phi_{1}$ was standardised so that

(a)

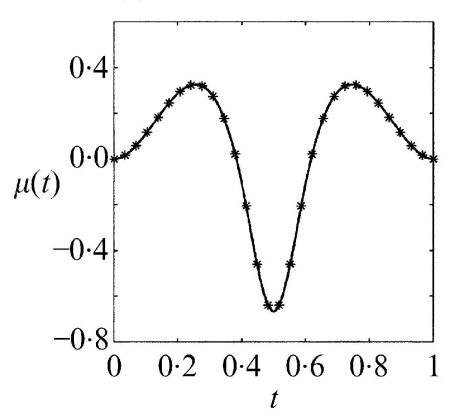

(b)

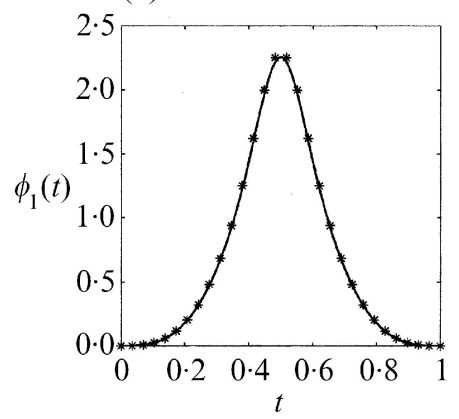

Fig. 2: Simulated models. (a) Mean function $\mu(t)$ and (b) amplitude variance component $\phi_{1}(t)$. Asterisks indicate function values at input grid points. 
$\sum_{j=1}^{m} \phi_{1}^{2}\left(t_{j}\right) / m=1$. The component scores $\left\{\xi_{i 1}\right\}$ followed a $\mathscr{N}\left(0, \lambda_{1}\right)$ distribution with $\lambda_{1}=0.75 \times 0 \cdot 10^{2}$. The errors $\left\{\varepsilon_{i j}\right\}$ had a $\mathscr{N}\left(0, \sigma^{2}\right)$ distribution with $\sigma=0 \cdot 10$ for the mean-plus-error model and $\sigma=0 \cdot 10 \sqrt{ } 0 \cdot 25$ for the variance-component model. Note that both models have the same overall amplitude variance, $E\left\{\sum_{j=1}^{m} Z^{2}\left(t_{j}\right)\right\}=0 \cdot 10^{2} m$, but differently split between systematic amplitude variability and random noise. For the variance-component model, $75 \%$ of the amplitude variance is associated with $\phi_{1}$. As warping model we took a piecewise cubic monotone function $g(t, \theta)$ with $\theta$ following a truncated normal distribution, with parameters $\theta_{0}=(0 \cdot 25,0 \cdot 50,0 \cdot 75)$, corresponding to the peaks and the trough of $\mu$, and $\tau=0.05 \times(1,1,1)$.

The maximum likelihood estimate was computed with the algorithm described in $\S 4$, which implicitly assumes that the mean-plus-error model is the correct one. For $g(t, \theta)$ we used the correct model and also two misspecified models: $\theta_{0}=(0 \cdot 25,0.75)$ and $\theta_{0}=0.50$, with the scale parameters always equal to 0.05. Explicitly, we consider the following scenarios.

Scenario 1 . The data are generated from the mean-plus-error model, so that the maximum likelihood estimator assumes the right amplitude variability and time-warping models.

Scenario 2. The data are generated from the variance-component model. The maximum likelihood estimator assumes the right warping model but the amplitude variability model is misspecified.

Scenario 3. The data are generated from the mean-plus-error model. The maximum likelihood estimator assumes the right amplitude variability model but a misspecified two-landmark model, corresponding to the peaks, is used.

Scenario 4. The data are generated from the mean-plus-error model. The maximum likelihood estimator assumes the right amplitude variability model but a misspecified one-landmark model, corresponding to the trough, is used.

\subsection{Comparison with landmark registration}

It is impossible to simulate proper landmark registration in this set-up, since it requires individual smoothing of the curves and careful identification of the landmarks, which cannot be done in a fully automated way. Therefore, we had to consider two simplified procedures, one that we call 'oracle' landmark registration and uses the actual realisations of $\theta$ as landmarks, and one that we call 'raw' landmark registration and uses the two local maxima and the minimum of the LOWEss smoother of the curves as landmarks. A properly implemented landmark registration will show an intermediate behaviour between these two simplified methods. For sampling Scenarios 3 and 4 we also considered two misspecified warping models, one that takes only the peaks as landmarks and one that takes only the trough as landmark.

Each sampling scenario was replicated 1000 times. As sample sizes we took $n=50$ and $n=100$, but the results were qualitatively similar, so we only report results for $n=50$. Simulated biases and root mean squared errors, as function of $t$, are shown in Fig. 3. The performance of the maximum likelihood estimator when both amplitude and warping components are well specified, Scenario 1, is comparable to that of oracle landmark registration. Misspecifying amplitude variability, Scenario 2, increases the variance of the maximum likelihood estimator but not the bias; in fact, the bias at the trough is smaller here than for Scenario 1. 


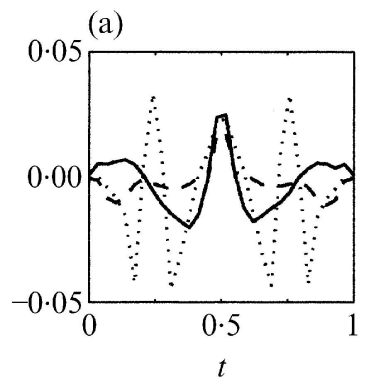

(e)

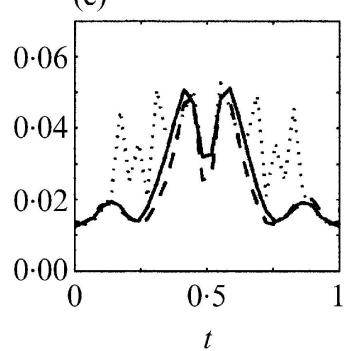

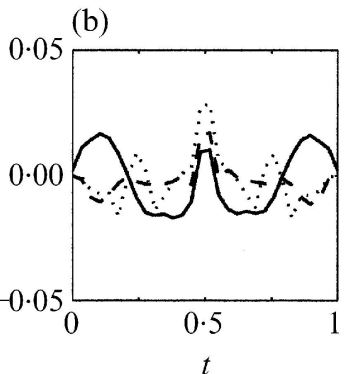

(f)

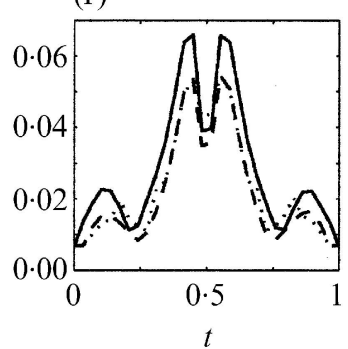

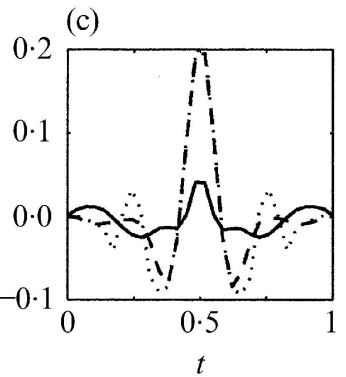

(d)
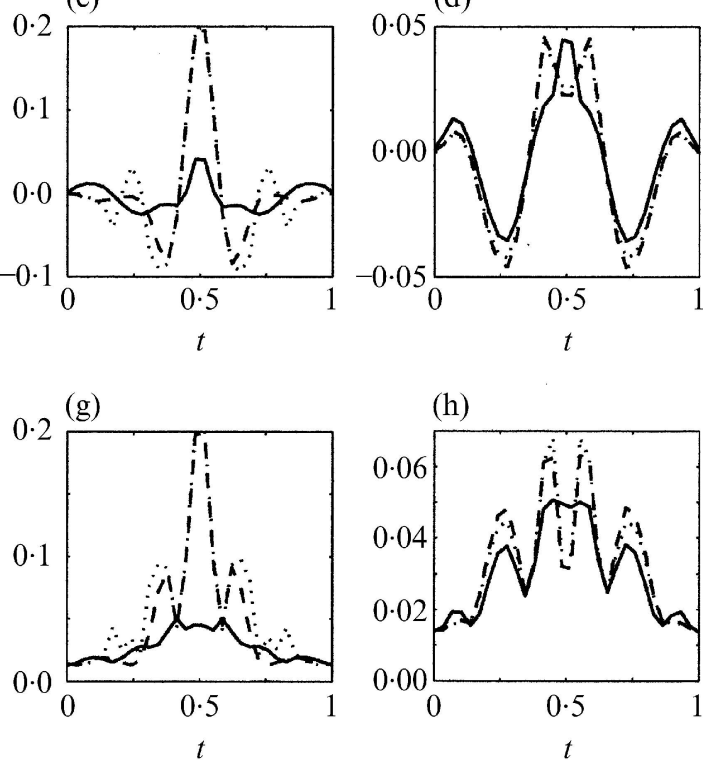

(h)

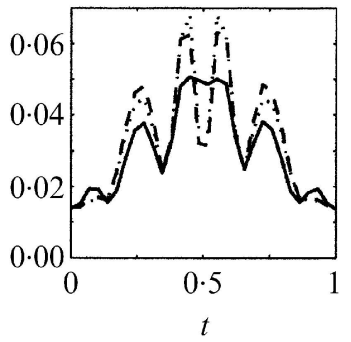

Fig. 3: Simulated models. Biases (a)-(d) and root mean squared errors (e)-(h) of maximum likelihood estimation, shown by solid lines, oracle landmark registration, dashed lines, and raw landmark registration, dotted lines, for Scenario 1 in (a) and (e), for Scenario 2 in (b) and (f), for Scenario 3 in (c) and (g), and for Scenario 4 in (d) and (h). Note the different scales for the vertical axes of (c) and (g).

The robustness of the maximum likelihood estimator to underspecification of landmarks is remarkable. As Fig. 3(c) shows, the bias of landmark registration at the trough is four times as large as the bias of the maximum likelihood estimator; it is practically as large as the bias of the cross-sectional mean, not shown. This behaviour has a simple explanation: the maximum likelihood estimator minimises a lack-of-fit criterion that, although not optimal for Scenarios 2-4, still penalises large shape deviations from the structural mean. On the other hand, landmark registration relies solely on the specified landmarks; since no lack-of-fit criterion is minimised, the method cannot compensate for missing landmarks, even when it is plain to see that the shape of the resulting estimator is not representative of the sample curves, as in Scenario 3.

\section{6·3. Comparison with continuous monotone registration}

Continuous monotone registration was proposed by Ramsay \& Li (1998) as a fully nonparametric alternative to landmark registration. This method does not require identification, or even existence, of landmarks. To compare maximum likelihood estimation with continuous monotone registration, we simulated the data using a warping model that is not associated with any landmarks. We took $g(t, \theta)$ such that

$$
\frac{\partial^{2} \log \left\{g^{-1}(t, \theta)\right\}}{\partial t^{2}}=\sum_{k=1}^{5} c_{k} \beta_{k}(t)
$$

with $\left\{\beta_{k}(t)\right\}$ cubic $B$-spline basis functions with equispaced knots in $[0,1]$ and $\left\{c_{k}\right\}$ independent and identically distributed coefficients following a $\operatorname{Un}(-1,1)$ distribution. 
(a)

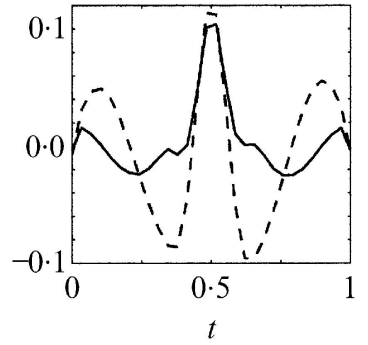

(e)

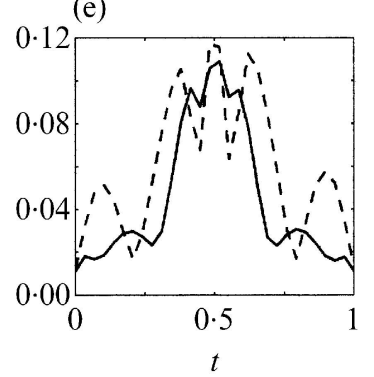

(b)

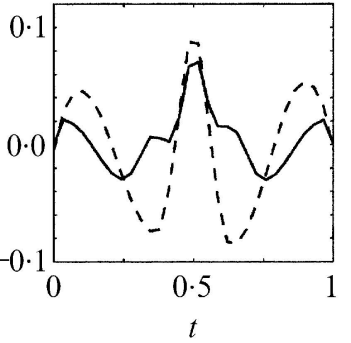

(f)

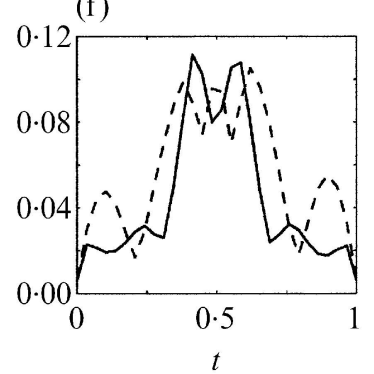

(c)

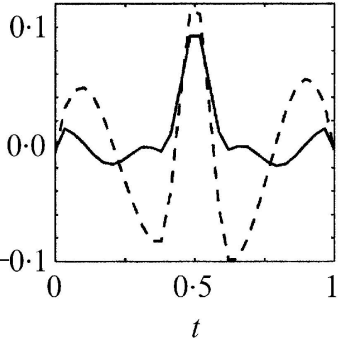

(g)

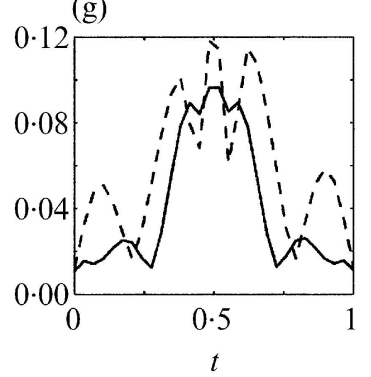

(d)

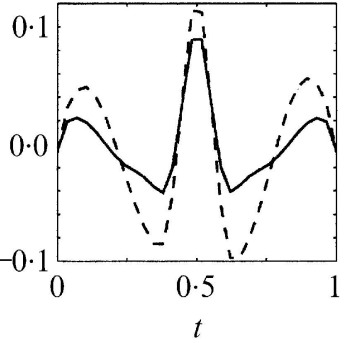

(h)

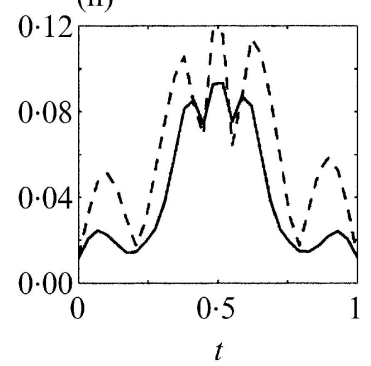

Fig. 4: Simulated models. Biases (a)-(d) and root mean squared errors (e)-(h) of maximum likelihood estimation, shown by solid lines, and continuous monotone registration, dashed lines, for Scenario 1 in (a) and (e), for Scenario 2 in (b) and (f), for Scenario 3 in (c) and (g), and for Scenario 4 in (d) and (h).

The same $B$-spline basis was used for registration, to avoid roughness penalisation of the warping functions and the consequent problem of choosing the smoothing parameter, which is excessively time consuming for this method. We used the software provided by James Ramsay in his website.

The raw data were generated from the same mean-plus-error model and a variancecomponent model as in $\S 6 \cdot 2$. However, since continuous monotone registration cannot be applied to raw data, we pre-smoothed the observations using penalised regression splines with 20 cubic $B$-spline basis functions with equispaced knots, choosing the smoothing parameter by generalised crossvalidation. The maximum likelihood estimator was computed on discretisations of these smooth curves on a grid of $m=30$ equispaced points. As regards the warping models assumed for maximum likelihood registration, we considered again the four Scenarios $1-4$ as in $\S 6 \cdot 2$. Each sampling situation was replicated 1000 times, with $n=50$ curves per sample.

The results are summarised in Fig. 4. Biases and mean squared errors were computed with $10 \%$ trimmed means because a few samples produced very outlying estimates for the continuous monotone registration method. We see that it makes little difference which warping model is used for the maximum likelihood estimator. This method outperforms continuous monotone registration in all scenarios. In particular, maximum likelihood provides much more accurate estimation than continuous monotone registration at the peaks. The explanation for this behaviour is that continuous monotone registration minimises a criterion that penalises misalignment at the trough much more strongly than misalignment at the peaks. In contrast, maximum likelihood estimation explicitly penalises misalignment at the peaks in Scenarios 1-3, thus providing better estimates even when the assumed warping models were not the true ones. 


\subsection{Confidence band coverage}

Finally, we ran some simulations to evaluate the finite-sample accuracy of the bootstrapped confidence intervals proposed in $\S 5$. Two hundred datasets were generated for Scenarios 1 and 2, with $n=50$ and $m=30$. Five hundred bootstrap samples were taken for each dataset and confidence bands of nominal level 90\% were constructed. Figure 5 shows the simulated coverage levels and average lengths. As expected, the coverage level deteriorates at the peaks and the trough, as usually happens with nonparametric estimators. Wild bootstrap intervals show a more stable coverage level around the nominal value, and, while they tend to be wider, in the present situation they seem to be preferable to model-based bootstrap bands. In models with more variance components, however, model-based bootstrap may produce wider confidence bands and have better coverage, as in the example shown in $\S 7$.
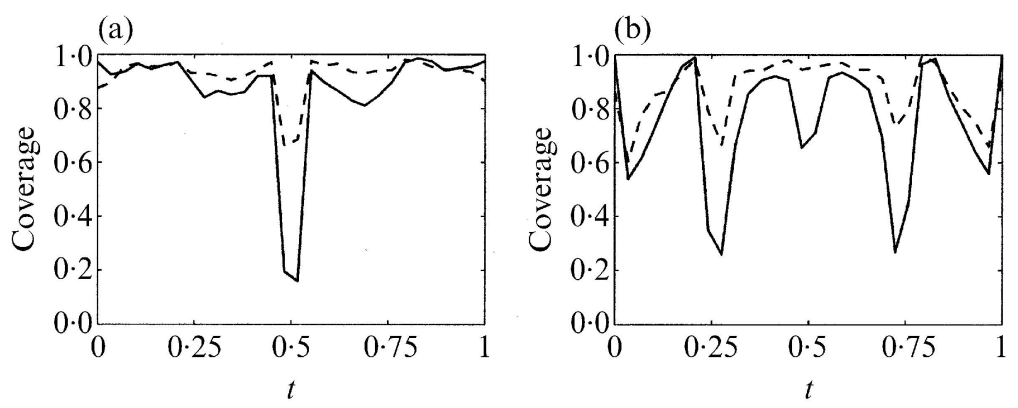

(c)
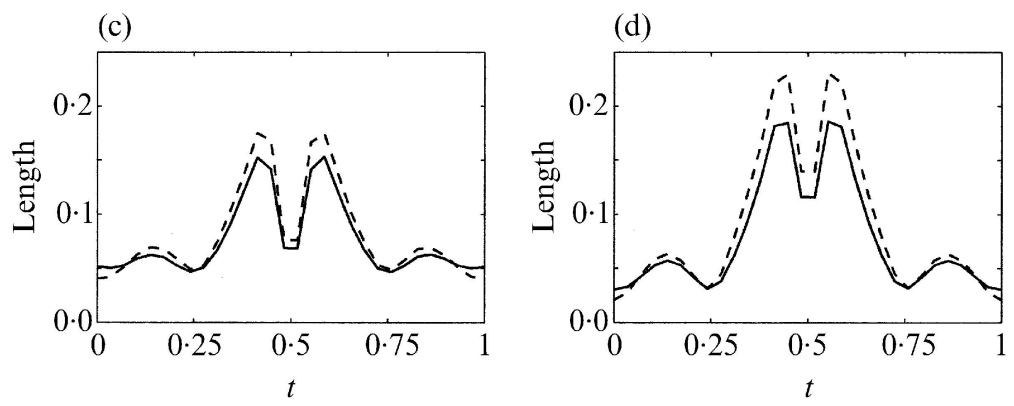

Fig. 5: Simulated data. Pointwise coverage levels and mean lengths of model-based, shown by solid lines, and wild, dashed lines, bootstrapped confidence bands for mean-plus-error model in (a) and (c), and for variancecomponent model in (b) and (d).

\section{Application: Analysis of human growth Data}

The First Zurich Growth Longitudinal Study produced a large number of datasets, consisting of measurements of different parts of the body taken from birth to adulthood. One of the goals of the researchers was to estimate the mean growth velocity curve, in order to characterise the growth spurts. Gasser et al. (1991) estimated individual velocity and acceleration curves using Gasser-Müller kernel smoothers, and computed landmark registration means using eight landmarks, namely the four zero crossings and the four local extrema observable in typical acceleration curves.

Here we report the analysis of leg-growth velocity from 3 to 21 years of age. We chose leg measurements because these curves have prominent mid-growth spurts, in addition to the 
well-known pubertal spurt. For girls, both spurts occur in close succession and are roughly of the same size, complicating the registration process. The observed data consisted of leg-length measurements taken annually from 3 to 9 years and biannually from then on. From these measurements we computed raw velocities by finite differentiation, taking the midpoints of age intervals as input grid. This yields a total of $m=29$ observations per person, for 112 girls and 120 boys.

The maximum likelihood estimate was computed using a two-dimensional warping model. Implicitly, we are interpreting $\theta$ as the location of the growth spurts. We tried several values of $\theta_{0}$ and $\tau$ and chose those that maximised the loglikelihood function: for girls, $\theta_{0}=(7,12)$ and $\tau=(1,1)$; for boys, $\theta_{0}=(7,14)$ and $\tau=(1,1)$. The maximum likelihood estimate was computed on an output grid of 100 equispaced points between 3.5 and 20.5 years.

For comparison, we also computed landmark registration means of smooth velocities, using the growth spurts as landmarks. The comparison is somewhat unfair with the maximum likelihood estimate, which is computed on much noisier raw velocities. Nevertheless, we can see in Fig. 6 that the maximum likelihood estimate is very close to the landmark registration mean for both sexes and, in particular, the mid-growth spurts are well determined.

Figure 6 also plots individual predictors $\left\{\hat{\theta}_{i 1}\right\}$ and $\left\{\hat{\theta}_{i 2}\right\}$ against growth spurt locations. The observed strong association supports our interpretation of the random effects $\theta_{i}$ as 'hidden landmarks'.

(a)

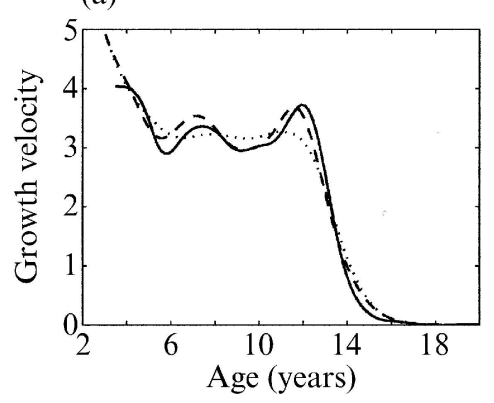

(c)

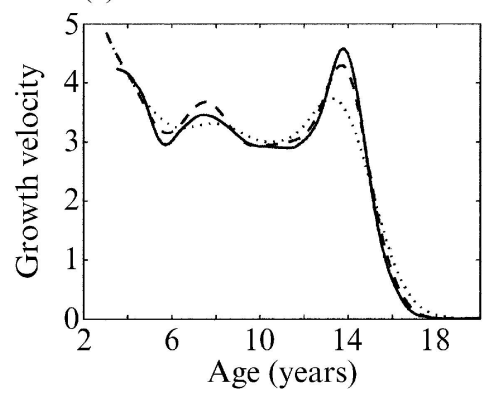

(b)

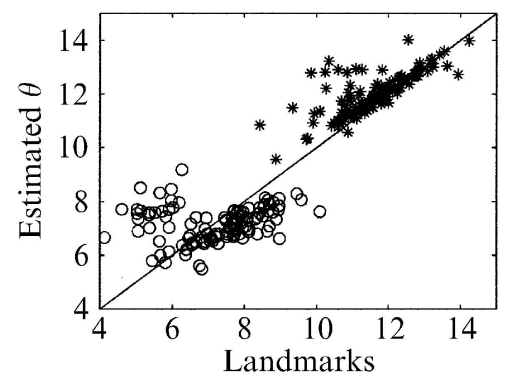

(d)

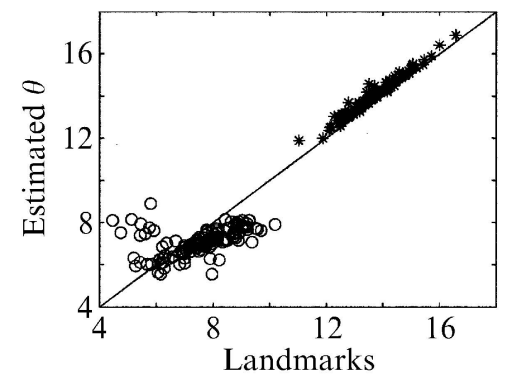

Fig. 6: Leg-growth data. Estimates of mean growth velocity in $\mathrm{cm} /$ year for girls (a) and boys (c) obtained by nonparametric maximum likelihood, shown by solid lines, landmark registration, dashed lines, and crosssectional mean, dotted lines. Scatter plots of mid-growth spurt location versus $\hat{\theta}_{1}$, shown by circles, and pubertal-spurt location versus $\hat{\theta}_{2}$, asterisks, for girls (b) and boys (d). 
We also obtained $90 \%$ confidence bands for the means, based on 1000 bootstrap samples for each method and each sex. As explained in $\S 5$, to apply model-based bootstrap the number of variance components in the model has to be determined. For each sex we estimated the 50 leading components and their variances, finding that the first six components explain, respectively, 23,16,15,14, 9 and 7 percent of the total variance for girls, and $24,15,13,11,8$ and 6 percent of the total variance for boys. We chose $q=4$ for both sexes, discarding those components that explain less than $10 \%$ of the amplitude variance. The resulting confidence bands are shown in Fig. 7 together with landmark registration means, which can be seen as the 'true means' in this example. We observe that modelbased bootstrap produces somewhat wider confidence bands than wild bootstrap and has better coverage. Among other things, a useful inference that can be drawn from the confidence bands is that the mid-growth spurt is a real structural feature of the growth process and not just an artifact of undersmoothing. Since most classical parametric models miss the growth spurt, its actual existence was debated in the early 80 's, when it was first detected and characterised by nonparametric methods.

(a)

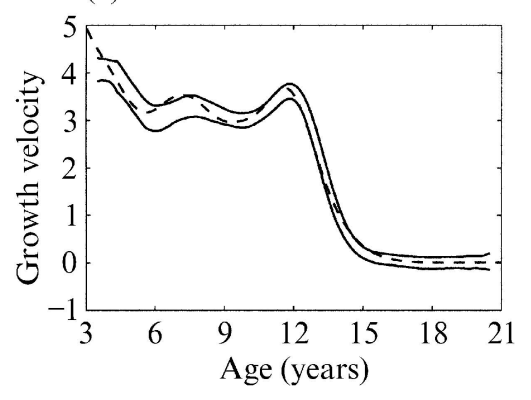

(c)

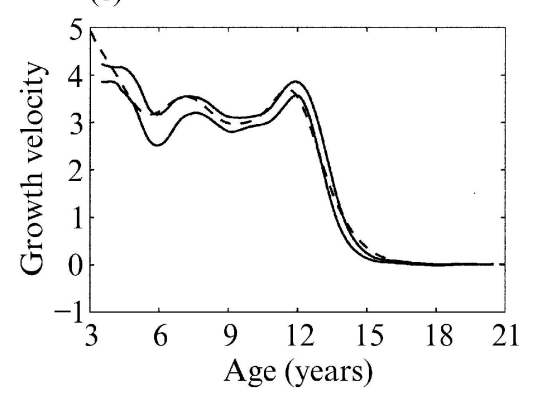

(b)

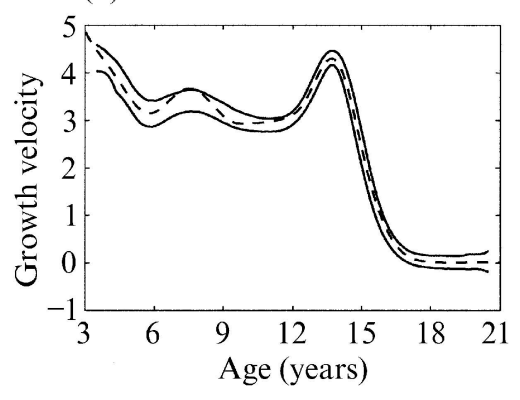

(d)

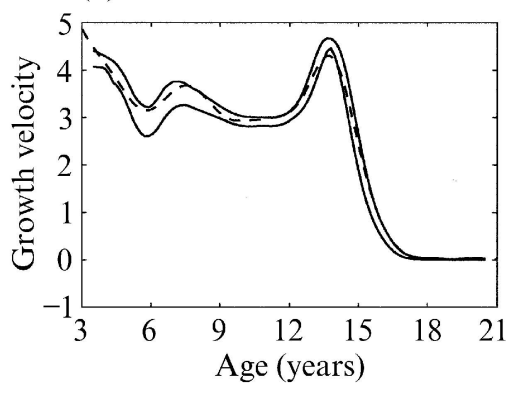

Fig. 7: Leg-growth data. Confidence bands of level 90\%, shown by solid lines, and landmark registration means, dashed lines, of leg-growth velocities in $\mathrm{cm} /$ year for girls shown in (a) and (c), and for boys in (b) and (d). Confidence bands obtained by model-based bootstrap in (a) and (b), and wild bootstrap in (c) and (d).

\section{Discussion}

The registration method proposed in this paper has a number of advantages over existing methodology. It does not require individual landmark identification or presmoothing of the data, and its implementation is considerably less time consuming and complicated than continuous monotone registration. As we see it, maximum likelihood 
registration combines appealing properties of both landmark and continuous monotone registration: like continuous monotone registration, it minimises a lack-of-fit criterion and is thus robust to misspecification of the warping model; like landmark registration, it explicitly models time variability at the salient features of the curves, which makes the warping model flexible and parsimonious at the same time.

We foresee a number of extensions and modifications of this method that can be more suitable for some particular situations. For instance, when the number of observations per curve is large and the data are very noisy, it may be worth considering spline models for $\mu$, rather than the full nonparametric approach of this paper. This will reduce the estimation problem to a more manageable finite-dimensional optimisation, and simultaneous estimation of the mean and the variance components may be less cumbersome. Of course, this would also introduce the problem of knot placement and selection, or roughness penalisation and selection of smoothing parameters, so more research is needed before we can make claims about the relative merits of each approach.

We also think that this method can be extended to fields of applications that require more complex warping models, such as image alignment, more easily than other registration methods. This is currently being investigated by the authors.

\section{ACKNOWLEDGEMENT}

This work was supported in part by grants from the Swiss National Science Foundation.

\section{APPENDIX}

Technical details about asymptotic results

We introduce some basic concepts on differentiation in functional spaces; a more detailed treatment, with applications to optimisation of functionals, is given in Luenberger (1969, Ch. 7). Let $\left(\mathscr{S}_{1},\|\cdot\|_{1}\right)$ and $\left(\mathscr{S}_{2},\|\cdot\|_{2}\right)$ be normed linear spaces and $F: \mathscr{S}_{1} \rightarrow \mathscr{S}_{2} ; F$ is said to be Fréchet differentiable at $a \in \mathscr{S}_{1}$ if there is a linear functional $D F(a): \mathscr{S}_{1} \rightarrow \mathscr{S}_{2}$ such that $\|F(a+b)-F(a)-D F(a) b\|_{2}=o\left(\|b\|_{1}\right)$. When $D F(a)$ is itself differentiable as a function of $a$ in the norm $\|D F(a)\|=\sup \left\{\|D F(a) b\|_{2}:\|b\|_{1} \leqslant 1\right\}, F$ is said to be twice Fréchet differentiable and the second differential is denoted by $D^{2} F(a)$. These definitions will be applied to $\mathscr{S}_{1}=\mathscr{M} \subseteq \mathscr{L}^{\infty}(T)$ equipped with the sup norm, $\|f\|=\sup _{t \in T}|f(t)|$, and $\mathscr{S}_{2}=\mathbb{R}$ with the usual absolute value as norm. For Theorem 1 we also need to define the tensor product of functionals: for each $h \in \mathscr{I}_{1}, F_{1} \otimes F_{2} h$ is defined as the functional $\left(F_{2} h\right) F_{1}$, and, for a pair $\left(h_{1}, h_{2}\right) \in \mathscr{S}_{1}^{2}, F_{1} \otimes F_{2}\left(h_{1}, h_{2}\right)=\left(F_{2} h_{1}\right)\left(F_{1} h_{2}\right)$.

Theorem A1. Given $x \in \mathbb{R}^{m}$, let $\ell_{x}(\mu)=\log f(x ; \mu)$. Then $\ell_{x}: \mathscr{L}^{\infty}(T) \rightarrow \mathbb{R}$ is twice Fréchet differentiable at every $\mu \in \mathscr{L}^{\infty}(T)$. The first differential is given by

$$
D \ell_{x}(\mu) h=\int k_{x}(s ; \mu) h(s) d s
$$

where

$$
k_{x}(s ; \mu)=\frac{1}{\sigma^{2}} \sum_{j=1}^{m}\left\{x_{j}-\mu(s)\right\} w_{j}(s \mid x ; \mu)
$$


and $w_{j}(s \mid x ; \mu)$ is the conditional density of $g\left(t_{j}, \theta\right)$ given $x$. The second differential is given by

$$
D^{2} \ell_{x}(\mu)\left(h_{1}, h_{2}\right)=\iint \rho_{x}(s, t) h_{1}(s) h_{2}(t) d s d t+\int \eta_{x}(s) h_{1}(s) h_{2}(s) d s-D \ell_{x}(\mu) \otimes D \ell_{x}(\mu)\left(h_{1}, h_{2}\right),
$$

where

$$
\begin{aligned}
\rho_{x}(s, t) & =\frac{1}{\sigma^{4}} \sum_{j=1}^{m} \sum_{\substack{k=1 \\
k \neq j}}^{m}\left\{x_{j}-\mu(s)\right\}\left\{x_{k}-\mu(t)\right\} v_{j k}(s, t \mid x ; \mu), \\
\eta_{x}(s) & =\frac{1}{\sigma^{4}} \sum_{j=1}^{m}\left[\left\{x_{j}-\mu(s)\right\}^{2}-\sigma^{2}\right] w_{j}(s \mid x ; \mu),
\end{aligned}
$$

and $v_{j k}(s, t \mid x ; \mu)$ is the joint conditional density of $\left(g\left(t_{j}, \theta\right), g\left(t_{k}, \theta\right)\right)$ given $x$.

Proof. We only need to show that $f(x ; \mu)$ is twice differentiable as a function of $\mu$ for each $x$, and it will follow that $D \ell_{x}(\mu)=D f(x ; \mu) / f(x ; \mu)$ and

$$
D^{2} \ell_{x}(\mu)=D^{2} f(x ; \mu) / f(x ; \mu)-\{D f(x ; \mu) \otimes D f(x ; \mu)\} / f^{2}(x ; \mu) .
$$

Given $x \in \mathbb{R}^{m}$, let $F_{x}(v)=\left(2 \pi \sigma^{2}\right)^{-m / 2} \exp \left(-\|x-v\|^{2} / 2 \sigma^{2}\right)$. This function is twice differentiable for every $v \in \mathbb{R}^{m}$, and the differentials are $D F_{x}(v)=F_{x}(v) \sigma^{-2}(x-v)^{\mathrm{T}}$ and

$$
D^{2} F_{x}(v)=F_{x}(v) \sigma^{-4}(x-v)(x-v)^{\mathrm{T}}-F_{x}(v) \sigma^{-2} I .
$$

Then the residuals $R_{x}^{(1)}(v, w)=F_{x}(v+w)-F_{x}(v)-D F_{x}(v) w$ and

$$
R_{x}^{(2)}(v, w)=D F_{x}(v+w)-D F_{x}(v)-D^{2} F_{x}(v) w
$$

are $o(\|w\|)$ for each $v$.

Now, since $f(x ; \mu)=\int F_{x}\left[\mu\left\{g\left(t^{*}, \theta\right)\right\}\right] f(\theta) d \theta$, it is not difficult to show that

$$
\begin{gathered}
D f(x ; \mu) h=\int D F_{x}\left[\mu\left\{g\left(t^{*}, \theta\right)\right\}\right] h\left\{g\left(t^{*}, \theta\right)\right\} f(\theta) d \theta, \\
D^{2} f(x ; \mu)\left(h_{1}, h_{2}\right)=\int h_{2}\left\{g\left(t^{*}, \theta\right)\right\}^{\mathrm{T}} D^{2} F_{x}\left[\mu\left\{g\left(t^{*}, \theta\right)\right\}\right] h_{1}\left\{g\left(t^{*}, \theta\right)\right\} f(\theta) d \theta .
\end{gathered}
$$

To prove this, note that

$$
f(x ; \mu+h)-f(x ; \mu)-D f(x ; \mu) h=\int R_{x}^{(1)}\left[\mu\left\{g\left(t^{*}, \theta\right)\right\}, h\left\{g\left(t^{*}, \theta\right)\right\}\right] f(\theta) d \theta
$$

and, since $\left\|h\left\{g\left(t^{*}, \theta\right)\right\}\right\| \leqslant \sqrt{ } m\|h\|$,

$$
\frac{|f(x ; \mu+h)-f(x ; \mu)-D f(x ; \mu) h|}{\|h\|} \leqslant \sqrt{ } m \int \frac{\left|R_{x}^{(1)}\left[\mu\left\{g\left(t^{*}, \theta\right)\right\}, h\left\{g\left(t^{*}, \theta\right)\right\}\right]\right|}{\left\|h\left\{g\left(t^{*}, \theta\right)\right\}\right\|} f(\theta) d \theta .
$$

By dominated convergence, the right-hand side goes to zero as $\|h\|$ goes to zero and then (A1) holds. For the second differential, we have that

$$
\left\{D f\left(x ; \mu+h_{1}\right)-D f(x ; \mu)-D^{2} f(x ; \mu) h_{1}\right\} h_{2}=\int h_{2}\left\{g\left(t^{*}, \theta\right)\right\}^{\mathrm{T}} R_{x}^{(2)}\left[\mu\left\{g\left(t^{*}, \theta\right)\right\}, h_{1}\left\{g\left(t^{*}, \theta\right)\right\}\right] f(\theta) d \theta
$$

and then

$$
\left\|D f\left(x ; \mu+h_{1}\right)-D f(x ; \mu)-D^{2} f(x ; \mu) h_{1}\right\| \leqslant \int\left\|R_{x}^{(2)}\left[\mu\left\{g\left(t^{*}, \theta\right)\right\}, h_{1}\left\{g\left(t^{*}, \theta\right)\right\}\right]\right\| f(\theta) d \theta .
$$

Again, this implies that $\left\|D f\left(x ; \mu+h_{1}\right)-D f(x ; \mu)-D^{2} f(x ; \mu) h_{1}\right\|=o\left(\left\|h_{1}\right\|\right)$ and then (A2) holds. 
The first and second differentials of $L_{n}(\mu)$ are $\Psi_{n}(\mu):=E_{n} D \ell_{x}(\mu)$ and $\dot{\Psi}_{n}(\mu):=E_{n} D^{2} \ell_{x}(\mu)$, respectively. The asymptotic versions of these functions are obtained by substituting $E_{n}$ with $E_{0}$, and will be respectively denoted by $\Psi_{0}$ and $\dot{\Psi}_{0}$. Being a maximum of $L_{n}, \hat{\mu}$ is a zero of $\Psi_{n}$ in the functional sense; that is, $\Psi_{n}(\hat{\mu}) h=0$ for all $h=\mathscr{M}$. Similarly, $\mu_{0}$ maximises $L_{0}$ and then $\Psi_{0}\left(\mu_{0}\right) h=0$ for all $h \in \mathscr{M}$, which can be verified by direct calculation.

Proof of Theorem 1. (i) First, note that $L_{0}$ has a unique maximum at $\mu_{0}$ : since $\log x \leqslant 2(\sqrt{ } x-1)$ for all $x \geqslant 0$, we have

$$
\begin{aligned}
L_{0}(\mu)-L_{0}\left(\mu_{0}\right) & =\int \log \left\{\frac{f(x ; \mu)}{f\left(x ; \mu_{0}\right)}\right\} f\left(x ; \mu_{0}\right) d x \\
& \leqslant 2\left\{\int \sqrt{ } f(x ; \mu) \sqrt{ } f\left(x ; \mu_{0}\right) d x-1\right\} \\
& =-\int\left\{\sqrt{ } f(x ; \mu)-\sqrt{ } f\left(x ; \mu_{0}\right)\right\}^{2} d x .
\end{aligned}
$$

Then $L_{0}(\mu)<L_{0}\left(\mu_{0}\right)$ whenever $\mu \neq \mu_{0}$, because the last integral is strictly negative for all $\mu \neq \mu_{0}$, by identifiability.

On the other hand, by Theorem 19.4 of van der Vaart (1998) we have $\sup _{\mu \in \mathscr{M}}\left|L_{n}(\mu)-L_{0}(\mu)\right| \rightarrow 0$ almost surely. This theorem applies because $\left|\ell_{x}\left(\mu_{1}\right)-\ell_{x}\left(\mu_{2}\right)\right| \leqslant \max _{\mu \in \mathscr{M}}\left\|D \ell_{x}(\mu)\right\|\left\|\mu_{1}-\mu_{2}\right\|$, and then the finiteness of the bracketing numbers required by this theorem follows from the compactness of $\mathscr{M}$.

In a compact space, almost sure uniform convergence of $L_{n}$ and uniqueness of the maximiser of $L_{0}$ imply strong consistency of $\hat{\mu}_{n}$. To see this, take a realisation $\left\{\hat{\mu}_{n}^{(\omega)}\right\}$ such that $\left\|\hat{\mu}_{n}^{(\omega)}-\mu_{0}\right\| \nrightarrow 0$; here $\omega$ denotes an element in the underlying probability space. By compactness of $\mathscr{M}$, there is a subsequence $\hat{\mu}_{n_{k}}^{(\omega)}$ that converges to certain $\mu^{*} \neq \mu_{0}$. For this subsequence we have $L_{0}\left(\hat{\mu}_{n_{k}}^{(\omega)}\right) \rightarrow L_{0}\left(\mu^{*}\right)$, and also $L_{n_{k}}^{(\omega)}\left(\mu_{0}\right) \leqslant L_{n_{k}}^{(\omega)}\left(\hat{\mu}_{n_{k}}^{(\omega)}\right)$; if $\omega$ were such that $\left\|L_{n}^{(\omega)}-L_{0}\right\| \rightarrow 0$, this would imply that $L_{0}\left(\mu_{0}\right) \leqslant L_{0}\left(\mu^{*}\right)$, contradicting the uniqueness of $\mu_{0}$ as maximiser of $L_{0}$. Therefore, $\left\|\hat{\mu}_{n}^{(\omega)}-\mu_{0}\right\| \nrightarrow 0$ implies that $\left\|L_{n}^{(\omega)}-L\right\| \nrightarrow 0$, and hence $\operatorname{pr}\left(\left\|\hat{\mu}_{n}-\mu_{0}\right\| \not 0\right)=0$.

(ii) Since $\Psi_{n}(\hat{\mu})=\Psi_{0}\left(\mu_{0}\right)=0$ in the functional sense, we can write $-\sqrt{ } n\left\{\Psi_{0}(\hat{\mu})-\Psi_{0}\left(\mu_{0}\right)\right\}=$ $\sqrt{ } n\left(\Psi_{n}-\Psi_{0}\right)\left(\mu_{0}\right)+r_{n}$ with $r_{n}=\sqrt{ } n\left(\Psi_{n}-\Psi_{0}\right)\left(\hat{\mu}-\mu_{0}\right)$. The first step of this proof is to show that $\left\|r_{n}\right\|=o_{P}(1)$. Let $\mathbb{G}_{n}=\sqrt{ } n\left(E_{n}-E_{0}\right)$ denote the empirical process. Then $\sqrt{ } n\left(\Psi_{n}-\Psi_{0}\right)\left(\hat{\mu}-\mu_{0}\right) h=$ $\mathbb{G}_{n} \psi_{\hat{\mu}, h}$, where $\psi_{\mu, h}(x)=\left\{D \ell_{x}(\mu)-D \ell_{x}\left(\mu_{0}\right)\right\} h$. The family $\left\{\psi_{\mu, h}:(\mu, h) \in \mathscr{M} \times \mathscr{M}\right\}$ is a Donsker class because it is Lipschitz in $(\mu, h)$, with a square-integrable Lipschitz factor, and the parameter space $\mathscr{M} \times \mathscr{M}$ is compact (van der Vaart, 1998, Theorem 19.5). Then, since $\hat{\mu} \rightarrow \mu_{0}$ in probability, we have that $\sup _{h \in \mathscr{M}}\left|\mathbb{G}_{n} \psi_{\hat{\mu}, h}\right| \rightarrow \sup _{h \in \mathscr{M}}\left|\mathbb{G}_{\mu_{0}, h}\right|$ in distribution, where $\mathbb{G}$ is a Gaussian element with zero mean and covariance

$$
E\left(\mathbb{G} \psi_{\mu_{1}, h_{1}} \mathbb{G} \psi_{\mu_{2}, h_{2}}\right)=E_{0}\left\{\psi_{\mu_{1}, h_{1}}(x) \psi_{\mu_{2}, h_{2}}(x)\right\}-E_{0}\left\{\psi_{\mu_{1}, h_{1}}(x)\right\} E\left\{\psi_{\mu_{2}, h_{2}}(x)\right\} .
$$

Since $\psi_{\mu_{0}, h} \equiv 0$ for all $h$, it follows that $\sup _{h \in \mathscr{M}}\left|\mathbb{G}_{n} \psi_{\hat{\mu}, h}\right| \rightarrow 0$ in distribution, which is just another way of writing $\left\|r_{n}\right\|=o_{P}(1)$.

We now find the limiting distribution of $\sqrt{ } n\left(\Psi_{n}-\Psi_{0}\right)\left(\mu_{0}\right)$. Again, we can write

$$
\sqrt{ } n\left(\Psi_{n}-\Psi_{0}\right)\left(\mu_{0}\right) h=\mathbb{G}_{n} \xi_{h},
$$

where $\xi_{h}(x)=D \ell_{x}\left(\mu_{0}\right) h$. As before, $\left\{\xi_{h}: h \in \mathscr{M}\right\}$ is a Donsker family, so that $\mathbb{G}_{n} \xi_{h} \rightarrow \mathbb{G} \xi_{h}$ in distribution, uniformly in $h$, where $\mathbb{G}$ is a zero-mean Gaussian element with covariances given by $E\left(\mathbb{G} \xi_{h_{1}} \mathbb{G} \xi_{h_{2}}\right)=E_{0}\left\{\xi_{h_{1}}(x) \xi_{h_{2}}(x)\right\}-E_{0}\left\{\xi_{h_{1}}(x)\right\} E_{0}\left\{\xi_{h_{2}}(x)\right\}=\mathscr{T} h_{1} h_{2}$. This together with $\left\|r_{n}\right\|=o_{P}(1)$ imply that $\sqrt{ } n\left\{\Psi_{0}(\hat{\mu})-\Psi_{0}\left(\mu_{0}\right)\right\}$ converges to a Gaussian random element with mean zero and covariance operator $\mathscr{T}$. Since $\dot{\Psi}_{0}\left(\mu_{0}\right)=E_{0}\left\{D^{2} \ell_{x}\left(\mu_{0}\right)\right\}=-\mathscr{T} \neq 0$, the functional delta method (van der Vaart, 1998, Theorem 20.8) applied to $\Psi_{0}^{-1}$ implies that $\sqrt{ } n\left(\hat{\mu}-\mu_{0}\right)$ converges in distribution to a Gaussian random element with mean zero and variance operator $\mathscr{T}^{-1}$. 


\section{REFERENCES}

Fritsch, F. N. \& Carlson, R. E. (1980). Monotone piecewise cubic interpolation. SiAM J. Numer. Anal. 17, 238-46.

Gasser, T. \& Kneip, A. (1995). Searching for structure in curve samples. J. Am. Statist. Assoc. 90, 1179-88.

Gasser, T., Kneip, A., Binding, A., Prader, A. \& Molinari, L. (1991). The dynamics of linear growth in distance, velocity and acceleration. Ann. Hum. Biol. 18, 187-205.

Gervini, D. \& Gasser, T. (2004). Self-modelling warping functions. J. R. Statist. Soc. B 66, 959-71.

KNeIP, A. \& Engel, J. (1995). Model estimation in nonlinear regression under shape invariance. Ann. Statist. 23, 551-70.

KNeIP, A. \& Gasser, T. (1992). Statistical tools to analyze data representing a sample of curves. Ann. Statist. 20, $1266-305$.

Kneip, A., Li, X., MacGibbon, K. B. \& Ramsay, J. O. (2000). Curve registration by local regression. Can. J. Statist. 28, 19-29.

Luenberger, D. (1969). Optimization by Vector Space Methods. New York: John Wiley.

Ramsay, J. O. (1988). Monotone regression splines in action. Statist. Sci. 3, 425-41.

Ramsay, J. O. (1998). Estimating smooth monotone functions. J. R. Statist. Soc. B 60, 365-75.

Ramsay, J. O. \& Li, X. C. (1998). Curve registration. J. R. Statist. Soc. B 60, 351-63.

Ramsay, J. O. \& Silverman, B. W. (1997). Functional Data Analysis. New York: Springer-Verlag.

Ramsay, J. O. \& Silverman, B. W. (2002). Applied Functional Data Analysis: Methods and Case Studies. New York: Springer-Verlag.

RønN, B. B. (2001). Nonparametric maximum likelihood estimation for shifted curves. J. R. Statist. Soc. B 63, 243-59.

SiLVERMAN, B. W. (1995). Incorporating parametric effects into functional principal components analysis. J. R. Statist. Soc. B 57, 673-89.

VAN DER VAart, A. W. (1998). Asymptotic Statistics. Cambridge University Press.

Wand, M. P. \& Jones, M. C. (1995). Kernel Smoothing. Boca Raton, FL: Chapman and Hall/CRC Press.

Wang, K. \& Gasser, T. (1999). Synchronizing sample curves nonparametrically. Ann. Statist. 27, 439-60.

[Received December 2004. Revised July 2005] 